\title{
CONVERGENCIA DE VARIABLES ALEATORIAS EN ESPACIOS DE HILBERT $\mathrm{L}_{2}(\Omega, A, P)$
}

Wilfredo Dominguez $C^{*}$

\section{RESUMEN}

Una variable aleatoria $\mathrm{X}$ es una función medible real valorada, cuyo dominio es el espacio muestral $\Omega$ y cuyo rango es un conjunto no vacío de números reales, es decir $\mathrm{X}$ es variable aleatoria si $\forall \mathrm{x} \in \mathrm{R}$, el suceso $\{\omega \in \Omega ; \mathrm{X}(\omega) \leq \mathrm{x}\} \in A$.

En el presente trabajo se extienden algunos resultados del caso univariado a la teoría de variables aleatorias complejas y su relación con los espacios de Hilbert.

\section{Introducción}

Formalmente una v.a. X es una función

$$
\mathrm{X}: \Omega \rightarrow \mathrm{R}_{\mathrm{X}} \subset \mathrm{R} \quad ; \quad \mathrm{R}_{\mathrm{X}} \neq \phi
$$

con la condición

$$
[\mathrm{X} \leq \mathrm{x}] \in A
$$

donde $A$ es un sigma álgebra tal que $A \subset \mathrm{P}(\Omega)$. Ahora si $A=\mathrm{P}(\Omega)$, la condición (1) se cumple inmediatamente, pues el suceso $[\mathrm{X} \leq \mathrm{x}]$ es un subconjunto de $\Omega$, el cual siempre es un suceso. Si $A \subset \mathrm{P}(\Omega)$ puede ocurrir que la condición (1) no se cumpla, para algún valor de $\mathrm{x}$, como consecuencia, la función $\mathrm{X}$ no sería variable aleatoria.

Una v.a. X se considera definida por completo, si se conoce $\omega$, el resultado del experimento. Así pues, la v.a. X en el espacio probabilístico $(\Omega, A, \mathrm{P})$, que describe el experimento aleatorio dado, es una función $\mathrm{X}(\omega)$ de un suceso aleatorio. Una condición equivalente a (1) es

$$
\{\omega ; \mathrm{X}(\omega) \in \Delta\} \in A
$$

donde $\Delta$ es un boreliano de R. Es decir X es una función medible. 
El ejemplo más simple de una v.a. X es la función indicadora, es decir

$$
\begin{array}{ll}
\mathrm{I}_{\mathrm{A}}(\omega)=1 & \text { si } \omega \in A \\
\mathrm{I}_{\mathrm{A}}(\omega)=0 & \text { si } \omega \notin A
\end{array}
$$

Teorema 1. Para que la función X sea P-medible es necesario y suficiente que para todo c $\in \mathrm{R}$, el conjunto

$$
\mathrm{B}=\{\omega ; \mathrm{X}(\omega)<\mathrm{c}\}
$$

sea P-medible; es decir $\mathrm{B} \in A$.

Demostración. La condición necesaria es directa, pues el intervalo $<-\infty$, c $>$ es un conjunto boreliano. Para probar la suficiencia hay que tener en cuenta que

$$
\bigcap_{n=1}^{\infty}\left[X<c+\frac{1}{2^{n}}\right]=[X \leq c] \quad \forall \mathrm{c} \in \mathbf{R}
$$

Teorema 2. El límite de una sucesión convergente $\left\{X_{n}\right\} ; n \geq 1$, de variables aleatorias es una función P-medible.

Demostración.- $\quad$ Sea $\lim \mathrm{X}_{\mathrm{n}}=\mathrm{X}$

$$
n \rightarrow \infty
$$

entonces $\quad\left\{\omega ; X_{m}(\omega)<c\right\}=\cup_{k} \cup_{n} \cap \underset{m>n}{\cap}\left\{\omega ; X_{m}(\omega)<c-1 / k\right\}$

Esto es cierto evidentemente, ahora si $\mathrm{X}(\omega)<\mathrm{c}$, entonces $\exists \mathrm{k} \in \mathrm{R}$ tal que:

Además para este $\mathrm{k}$ se puede escoger un $\mathrm{n}$ lo suficientemente grande para que $\mathrm{m} \geq \mathrm{n}$ y se cumpla la desigualdad

$$
X_{m}<c-\frac{1}{k}
$$

lo cual significa que $\omega$ figura en el lado derecho de la igualdad de (2). 
Por el contrario, si $\omega$ pertenece al lado derecho de (2), existe un $\mathrm{k}$ tal que para todos los $\mathrm{m}$ suficientemente grandes

$$
X_{m}(\varpi)<c-\frac{1}{k}
$$

Luego $\mathrm{X}(\omega)<\mathrm{c}$, es decir, $\omega$ figura en el lado izquierdo de (2).

Si las funciones $\mathrm{X}_{\mathrm{n}}(\omega)$ son medibles, entonces los conjuntos:

$$
\left\{\varpi ; X_{m}(\varpi)<c-\frac{1}{k}\right\}
$$

pertenecen a $A$, lo cual demuestra que $\mathrm{X}(\omega)$ es medible.

\section{PRELIMINARES}

Definición 1. Sea $\mathrm{H}$ un espacio vectorial complejo, cuyos elementos son variables aleatorias complejas definidas en un espacio muestral $\Omega$. Un producto interno en $\mathrm{H}$ es una aplicación $<,>: \mathrm{HxH} \rightarrow \mathrm{C}$ que asocia a cada par de variables aleatorias $\mathrm{X}_{1}, \mathrm{X}_{2}$ un escalar denotado por $<\mathrm{X}_{1}, \mathrm{X}_{2}>$, que cumple:
1) $<X_{1}, X_{2}>\geq 0 \quad \forall X_{1}, X_{2} \in H \quad y \quad<X_{1}, X_{1}>=0 \leftrightarrow X_{1}=0$
2) $<X_{1}+X_{2}, X_{3}>=<X_{1}, X_{3}>+<X_{2}, X_{3}>$
3) $<k X_{1}, X_{2}>=k<X_{1}, X_{2}>\forall X_{1}, X_{2} \in H \quad y \forall k \in C$
4) $<X_{1}, X_{2}>=<\overline{X_{2}}, \overline{X_{1}}>, \quad \forall X_{1}, X_{2} \in H$

Si $\mathrm{X}_{1}$ y $\mathrm{X}_{2}$ son dos variables aleatorias con funciones de densidad de $\mathrm{f}_{\mathrm{X} 1}(\mathrm{x})$ y $\mathrm{g}_{\mathrm{X} 2}(\mathrm{x})$ respectivamente entonces:

$$
\begin{aligned}
& <f_{X 1}(x), g_{X 2}(x)>=\int_{a}^{b} f_{X 1}(x) g_{X 2}(x) d x \\
& X(\varpi)<c-\frac{1}{k}
\end{aligned}
$$

es un producto interno de $\mathrm{f} y \mathrm{~g}$. Se puede verificar inmediatamente que (3) cumple las condiciones exigidas de un producto interno. 
Definición 2. Sea $(\mathrm{H} ;<,>)$ un espacio producto interno con la norma

$$
\|\|=\left[<\mathrm{X}_{1}, \mathrm{X}_{2}>\right]^{1 / 2}
$$

entonces $(\mathrm{H},<,>)$ se llama espacio de variables aleatorias de Hilbert; el cual será denotado por $\mathscr{L}_{2}(\Omega, A, \mathrm{P})$

Definición 3. Sean $\mathrm{X}_{1}, \mathrm{X}_{2}, \mathrm{X}_{3}, \ldots .$. una sucesión de variables aleatorias discretas dadas por :

$$
X_{n}=\frac{k}{n}, \text { si } \frac{k}{n} \leq X_{n}<\frac{k+1}{n} \quad, \quad k=0, \pm 1, \pm 2, \ldots
$$

$\mathrm{Si} \mathrm{E}\left(\mathrm{X}_{\mathrm{n}}\right)>\infty$ para cierto $\mathrm{n}$, existirá para todos los $\mathrm{n}, \mathrm{y}$, además, existe el límite

$$
\lim _{n \rightarrow \infty} E\left(X_{n}\right)=\lim _{n \rightarrow \infty} \sum_{k=-\infty}^{\infty} \frac{k}{n} P\left(\frac{k}{n} \leq X \leq \frac{k+1}{n}\right)
$$

este límite se llama esperanza matemática de la v.a. X.

Definición 4. Si $\mathrm{F}_{\mathrm{X}}(\mathrm{x})$ es una función de Distribución Acumulada de la variable aleatoria X, entonces

$$
E(X)=\int_{-\infty}^{\infty} x d F_{X}(x) \text {, cuando } \int_{-\infty}^{\infty}|x| d F_{X}(x)<\infty
$$

Ambas integrales son de stieltjes y se calculan como los límites de las sumas integrales.

Si existe la función de densidad $\mathrm{f}_{\mathrm{X}}(\mathrm{x})$, entonces

$$
E(X)=\int_{-\infty}^{\infty} x f_{X}(x) d x \text {, cuando } \int|x| f_{X}(x) d x<\infty
$$

\section{CONVERGENCIA DE VARIABLES ALEATORIAS EN ESPACIOS DE HILBERT}

Definición 5. Un conjunto $\Gamma$ de variables aleatorias con valores complejos, definidas en un espacio de probabilidad $(\Omega, A, \mathrm{P})$, con segundo momento finito $\mathrm{E}\left(|\mathrm{X}|^{2}\right)<\infty$ forma un espacio lineal normado de Hilbert $\mathscr{L}_{2}(\Omega, A, \mathrm{P})$ con el producto escalar

$$
<X_{1}, X_{2}>=E\left(X_{1} \overline{X_{2}}\right)
$$

y la norma

$$
\left\|X_{1}\right\|=\left[E\left|X_{1}\right|^{2}\right]^{1 / 2}
$$


Con la norma se determina la distancia entre las variables aleatorias de $\mathscr{L}_{2}(\Omega ; A, \mathrm{P})$

$$
d\left(X_{1}, X_{2}\right)=\left\|X_{1}-X_{2}\right\|
$$

Las variables aleatorias $\mathrm{X}_{1}, \mathrm{X}_{2}$ de $\mathscr{L}_{2}(\Omega, A, \mathrm{P})$ se denominan variables aleatorias en un espacio de Hilbert.

La sucesión de variables aleatorias $X_{n}, n=1,2,3, \ldots$, converge hacia la variable aleatoria $X$ con probabilidad 1 , si para la sucesión seleccionada $X_{n}(\omega), n=1,2,3, \ldots \ldots$

$$
\lim _{n \rightarrow \infty} X_{n}(\varpi)=X(\varpi)
$$

y para cualquier resultado elemental $\omega$, excepto de algunos resultados $\omega$ que tienen en su conjunto la probabilidad 0 .

Teorema 3. Sea la sucesión $\left\{X_{n}\right\}_{n \geq 1}$ de variables aleatorias en un espacio de Hilbert, se dice que esta sucesión converge en su media cuadrática hacia la variable aleatoria $\mathrm{X}$ con probabilidad 1, si

$$
\sum_{n=1}^{\infty}\left\|X_{n}-X\right\|^{2}<\infty
$$

Demostración. Para cualquier $\varepsilon>0$ en el suceso $A_{n}=\left\{\left|X_{n}(\omega)-X(\omega)\right|>\varepsilon\right\}$ y según la desigualdad de Chebishev se tiene que:

$$
P\left(A_{n}\right) \leq \frac{\left\|X_{n}-X\right\|^{2}}{\varepsilon^{2}}
$$

y en consecuencia

$$
\sum_{n=1}^{\infty} P\left(A_{n}\right) \leq \frac{1}{\varepsilon^{2}} \sum_{n=1}^{\infty}\left\|X_{n}-X\right\|^{2}<\infty
$$

Según el lema de Borel-Cantelli ocurre, con probabilidad 1, excepto solamente en un número finito de sucesos $A_{n}, n=1,2,3, \ldots$, lo cual significa que

$$
\lim _{n \rightarrow \infty} X_{n}(\varpi)=X(\varpi)
$$

con probabilidad 1 . 
La distancia en media cuadrática entre dos variables aleatorias en un espacio de Hilbert se define como la norma de la diferencia entre ellas, es decir

$$
\left\|X_{1}-X_{2}\right\|=\sqrt{E\left|X_{1}-X_{2}\right|^{2}}
$$

el cual es la distancia entre dos elementos en un espacio de Hilbert.

Esta distancia cumple la desigualdad triangular: para tres variables aleatorias $\mathrm{X}_{1}, \mathrm{X}_{2}, \mathrm{X}_{3}, \in$ $\mathrm{H}$, la distancia entre $\mathrm{X}_{1} \mathrm{y}_{2}$ no supera a la suma de las distancias entre $\mathrm{X}_{1} \mathrm{y} \mathrm{X}_{3}, \mathrm{y} \mathrm{X}_{2} \mathrm{y} \mathrm{X}_{3}$, es decir

$$
\left\|X_{1}-X_{2}\right\| \leq\left\|X_{1}-X_{3}\right\|+\left\|X_{2}-X_{3}\right\|
$$

El concepto de esperanza matemática se puede extender a variables aleatorias $\mathrm{X}$ que toman valores complejos:

$$
X_{j}=X_{j a}+i X_{j b}
$$

donde $\mathrm{X}_{\mathrm{ja}} \mathrm{y} \mathrm{X}_{\mathrm{jb}}$ son variables aleatorias reales. En este caso

$$
E\left(X_{j}\right)=E\left(X_{j a}\right)+i E\left(X_{j b}\right)
$$

Se puede demostrar que todas las propiedades de la esperanza matemática en $\mathrm{R}$ también se cumplen en el caso complejo.

En (7) se define el segundo momento como el valor de la media cuadrática.

La desigualdad de Cauchy-Bunjakovsky es dada por

$$
E\left(\left|X_{1} X_{2}\right|\right) \leq \sqrt{E\left(X_{1}^{2}\right)} \sqrt{E\left(X_{2}^{2}\right)}
$$

para cualquier par de variables aleatorias complejas $\mathrm{X}_{1}, \mathrm{X}_{2}$ que tienen segundo momento finito.

Teorema 4. La sucesión de variables aleatorias complejas $X_{n}, n=1,2,3, \ldots$ en un espacio de Hilbert $\mathrm{H}$ converge en media cuadrática hacia la v.a. X si y sólo si

$$
\lim _{n, m \rightarrow \infty}\left\|X_{n}-X_{m}\right\|=0
$$


Demostración. La necesidad del límite (8) se deduce de la desigualdad triangular: si la sucesión $\mathrm{X}_{\mathrm{n}}, \mathrm{n}=1,2,3, \ldots$, converge en media cuadrática hacia la variable aleatoria $\mathrm{X}$, entonces:

$$
\left\|X_{n}-X_{m}\right\| \leq\left\|X_{n}-X\right\|+\left\|X_{m}-X\right\| \rightarrow 0 \quad \text { para } n, m \rightarrow \infty
$$

La prueba de que para la condición (8) existe la variable aleatoria $X \in H$ tal que $\lim _{n \rightarrow \infty} X_{n}$ $=\mathrm{X}$ es una aplicación de la teoría de la medida.

Observemos que la sucesión $\mathrm{X}_{\mathrm{n}}, \mathrm{n}=1,2,3, \ldots$ que converge en media cuadrática hacia la variable aleatoria $\mathrm{X}$, estas variables aleatorias complejas tienen esperanzas finitas y se verifica

verificamos que

$$
E\left|X_{n}\right| \leq \sqrt{E\left(X_{n}^{2}\right)}<\infty
$$

$$
\lim _{n \rightarrow \infty} E\left(X_{n}\right)=E(X)
$$

En efecto, como ya se indicó, la v.a. $X \in H$ tiene una esperanza matemática finita $E(X)$ y también

$$
\left|E\left(X_{n}\right)-E(X)\right| \leq E\left|X_{n}-X\right| \leq \sqrt{E\left|X_{n}-X\right|^{2}} \rightarrow 0
$$

\section{REFERENCIAS}

1. Chumpitaz M. “Análisis Funcional”, U.N.I. 1984.

2. Chumpitaz M. "Medida e integración" U.N.I. 1989

3. Edwards R. E. "Theory of Random Measures on Locally Compact Spaces" Acta Math. Vol. 89, p. 160, 1963.

4. Parzen E. "Teoría moderna de Probabilidades y sus aplicaciones" Limusa 1979.

5. Rudin W. "Análisis Real y Complejo”, Editorial Alhambra 1979. 